\section{Clinical features and prognostic factors of Klebsiella endophthalmitis- 10-year experience in an endemic region}

\begin{abstract}
Aims To identify the clinical features and prognostic factors of endogenous endophthalmitis caused by Klebsiella pneumoniae.
\end{abstract}

Methods This is a retrospective case series of all patients with Klebsiella endophthalmitis managed from January 2006 to December 2015 by Kowloon East Ophthalmic Service. Statistical analysis involved hypothesis testing on the SPSS $\mathbf{1 8 . 0}$ software (SPSS). A significance level of $P<0.05$ was taken.

Results In the 10-year period, K. pneumoniae accounted for 19 out of 39 cases of endogenous endophthalmitis $(48.7 \%)$. The mean age of patients was 67.9 years. Bilateral involvement occurred in five patients $(26.3 \%)$. More than half of the patients $(10 / 19,52.6 \%)$ had underlying diabetes mellitus. Most patients had concurrent liver abscess (18/19, $94.74 \%)$. Ten patients $(52.6 \%)$ had disseminated intravascular coagulopathy. Eight patients $(42.1 \%)$ were in shock. The overall mortality was $21.1 \%(4 / 19)$. Septic shock was associated with a significantly higher mortality (50.0 vs $0 \%, P=0.018)$.

Among the 15 survivors, nine patients (60.0\%) required evisceration and three patients $\mathbf{2} 20.0 \%)$ had no light perception in an involved eye. Eyes with diffuse posterior involvement were less likely to have a final visual acuity of $\log$ MAR 0.30 or better than those with focal posterior involvement $\mathbf{( 4 . 7 6}$ vs $100 \% 4.76 \%, P=0.002)$. Patients with hypopyon were more likely to require

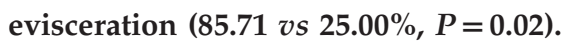
Conclusions Klebsiella endophthalmitis is associated with a high incidence of diabetes mellitus and liver abscess. Prognosis remains poor. Universal ocular screening and systemic
CY Chung $1,2,3$, ES Wong ${ }^{4}$, CCH Liu'1,2,3, MOM Wong ${ }^{4}$ and KKW Li $\mathrm{Li}^{1,2,3}$

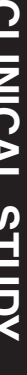

control in patients with Klebsiella sepsis are recommended.

Eye (2017) 31, 1569-1575; doi:10.1038/eye.2017.92; published online 16 June 2017

\section{Introduction}

Endogenous endophthalmitis is caused by the haematogenous spread of a microorganism from an infective focus across the blood-ocular barrier resulting in severe intraocular inflammation. Regional variations exist in the epidemiology of the causative microorganism. In North America and Europe, it is commonly caused by grampositive bacteria and fungi. However in East Asia, the microbiological spectrum is dominated by gram-negative bacteria, especially Klebsiella pneumoniae. ${ }^{1-4}$

For K. pneumoniae, endophthalmitis is only part of an invasive syndrome characterized of multifocal metastatic infection. Liver abscesses caused by K. pneumoniae were first described in the 1980s. ${ }^{5-7}$ Extrahepatic foci include urinary tract infection, empyema, peritonitis, splenic abscess, meningitis, musculoskeletal, and soft tissue infection. ${ }^{8}$

The increasing incidence of metastatic infection by K. pneumoniae in the East Asia in the recent decade may be explained by the emergence of a hypervirulent strain of Klebsiella, exemplified by its hypermucoviscous property. ${ }^{8}$ Known for its poor visual prognosis, endogenous endophthalmitis caused by K. pneumoniae still poses management challenges for the ophthalmologist. The management guidelines from the Endophthalmitis Vitrectomy Study, which focused on postoperative endophthalmitis, may not be directly applicable. ${ }^{3,9}$
${ }^{1}$ Department of Ophthalmology, United Christian Hospital, Kowloon, Hong Kong

${ }^{2}$ Department of Ophthalmology, Tseung Kwan O Hospital, New Territories, Hong Kong

${ }^{3}$ Department of Ophthalmology, LKS Faculty of Medicine, The University of Hong Kong, Hong Kong

${ }^{4}$ Hong Kong Eye Hospital, Kowloon, Hong Kong

\section{Correspondence:}

CY Chung, Department of Ophthalmology, United Christian Hospital, 130 Hip Wo Street, Kwun Tong, Kowloon, Hong Kong Tel: +852 39493411; Fax: +852 39495626 . E-mail: chungchungyee@ gmail.com

Received: 11 October 2016 Accepted in revised form: 8 April 2017 Published online: 16 June 2017 
This study, therefore, aimed to identify the systemic and ophthalmic clinical features of endogenous endophthalmitis caused by K. pneumoniae. Prognostic factors related to visual outcome and mortality were investigated. We also aimed to make recommendations on the management of Klebsiella endophthalmitis.

\section{Methods}

This is a retrospective case series of all patients with endogenous endophthalmitis due to K. pneumoniae managed from January 2006 to December 2015 by Kowloon East Ophthalmic Service, a tertiary eye center serving a population of $\sim 1$ million. The service is provided through United Christian Hospital and Tseung Kwan O Hospital. The study was approved by the Research Ethics Committee of the Hospital Authority (KCKE-11-0098/ER-3) and firmly adherent to the tenets of the Declaration of Helsinki.

\section{Subjects}

The cases were retrieved with the diagnosis of 'endophthalmitis' (code 360.00) from the electronic Clinical Management System of the Hospital Authority, Hong Kong.

The diagnosis of endogenous endophthalmitis caused by K. pneumoniae was defined as severe intraocular inflammation in a patient with clinical sepsis, with a positive microbiological culture of K. pneumoniae, especially from the vitreous/aqueous sample or blood sample. Patients with a potential exogenous cause of endophthalmitis, including those with recent eye injury, corneal ulcer or keratitis, previous glaucoma filtration surgery, and those a history of any intraocular surgery in the past 6 months were excluded from the study.

\section{Intervention}

The patients were arranged for systemic sepsis workup including of complete blood counts, clotting time, erythrocyte sedimentation rate, liver and renal function tests, microbiological culture of the peripheral blood, urine, and other samples if appropriate. Chest X-ray and abdominal imaging with ultrasonography and/or computed tomography were arranged to look for other infective foci.

All patients were administered intravenous antibiotics, the regime of which was continuously reviewed and adjusted by the attending ophthalmologist. Intensive care was offered to patients with haemodynamic instability. Drainage of infective foci was performed if appropriate. Ophthalmological interventions, including vitreous/ aqueous tapping for microbiological culture, intravitreal injection of antibiotics, were arranged on an urgent basis. Reinjection of intravitreal antibiotics and/or pars plana vitrectomy was performed according to the patients' clinical progress. Evisceration or enucleation was performed for those with a painful blind eye.

\section{Outcome of interest}

Demographic and clinical data concerning the patients' ophthalmic and systemic condition pertaining to the sepsis episode were collected from the relevant medical records. The primary outcome of interest was the visual outcome, including the visual acuity on the last follow-up and the proportion of patients requiring evisceration and/or enucleation. The secondary outcome of interest was mortality from the sepsis episode.

\section{Statistical analysis}

The visual acuity was measured on the Snellen's charts and converted to the logarithm of minimal angle of resolution (logMAR) units for analysis. The following $\log$ MAR denotations were used for non-numerical visual acuities: finger count $=1.7 \log \mathrm{MAR}$, hand movement $(\mathrm{HM})=2.0 \log \mathrm{MAR}$, light perception $(\mathrm{LP})=2.3 \log \mathrm{MAR}$, and no light perception (NLP) $=3.0 \log \mathrm{MAR} .{ }^{10}$ The visual acuity was defined as NLP after evisceration or enucleation. For patients with bilateral involvement of endogenous endophthalmitis, the visual acuity of the presenting eye was used for statistical analysis.

All statistical analysis was performed on the SPSS 18.0 software (SPSS, Chicago, IL, USA). Descriptive statistics, student $t$-test for continuous variables and $\chi^{2}$-test or Fisher's exact test for categorical variables were performed where appropriate. For $t$-test, equal variances between the groups were checked. The factors affecting mortality and the final visual outcome were analyzed. A significance level of $P<0.05$ was taken. Regression analysis was not performed due to the rarity of the disease of interest.

\section{Results}

In the study period, there were 39 cases of endogenous endophthalmitis, in which 19 cases were caused by K. pneumoniae. The mean duration of follow-up was 18.45 months (range 1 week to 102 months). The clinical profile of the patients is shown in Table 1.

\section{Patient profile}

The mean age of patients was $67.89 \pm 10.16$. There was a slight predominance for the male gender, with 12 male patients $(63.2 \%)$ and seven female patients $(36.8 \%)$. 


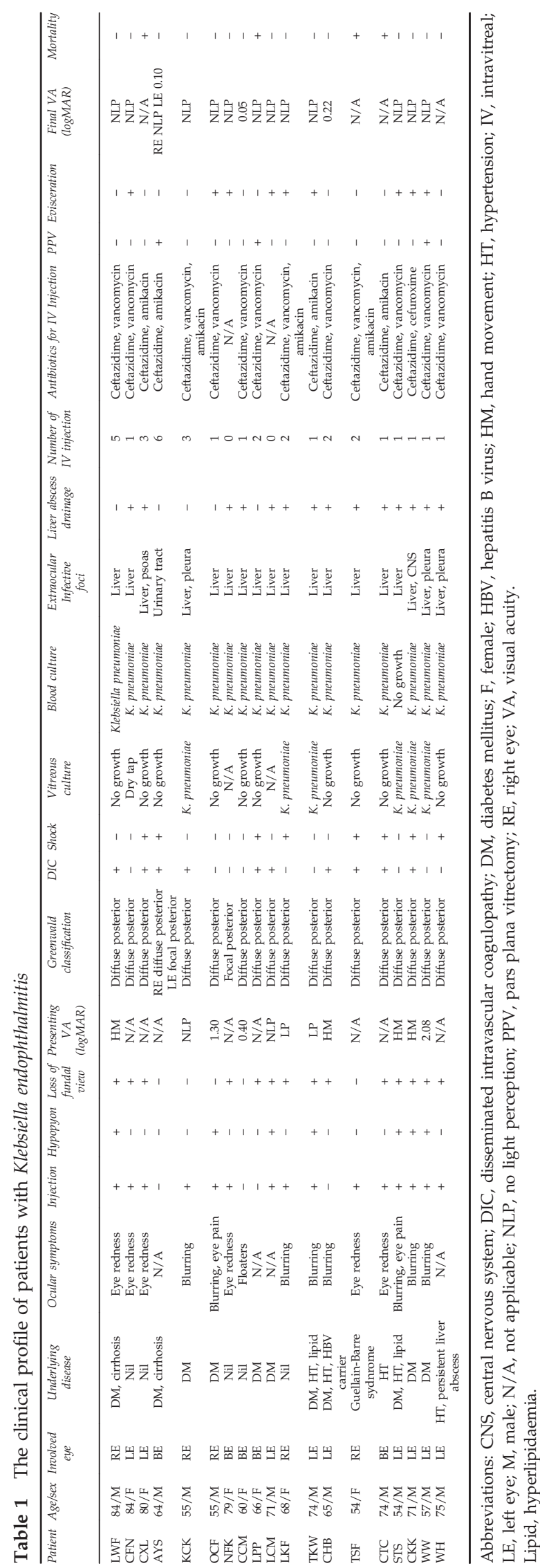

The left eye was involved in nine patients $(47.4 \%)$, the right eye involved in five patients $(26.3 \%)$ and both eyes were involved in five patients $(26.3 \%)$.

Ten patients $(52.6 \%)$ had underlying diabetes mellitus. Only three patients $(15.8 \%)$ had underlying liver cirrhosis or viral hepatitis. One patient had an inadequately treated liver abscess 2 months before the onset of Klebsiella endophthalmitis.

\section{Patient presentation}

All patients with Klebsiella endophthalmitis presented to the hospital with sepsis exemplified by a history of fever. At the time of ophthalmic consultation, only 10 patients $(52.6 \%)$ remained febrile.

Concerning the ocular presentation of endophthalmitis, nine patients $(47.4 \%)$ complained of blurring of vision. Conjunctival injection was observed in 15 patients $(78.9 \%)$, and hypopyon was observed in seven patients $(36.8 \%)$. The mean duration of ocular symptoms before the first ophthalmic consultation was $6.00 \pm 7.89$ days. Among the 11 patients who were conscious at the time of ophthalmic consultation, the median presenting visual acuity was HM (range NLP to logMAR 0.40).

Nevertheless, sepsis was the predominant clinical presentation of patients with Klebsiella endophthalmitis -none of them presented first with ocular symptoms.

All patients except two $(89.5 \%)$ had leukocytosis on blood test. Ten patients $(52.6 \%)$ had disseminated intravascular coagulopathy, defined as widespread pathological coagulation in the microvasculature, with the consumption of platelets and clotting factors from the systemic circulation. On blood tests, these patients had thrombocytopenia and prolongation of prothromin time and/or activated partial thromboplastin time. Eight patients $(42.1 \%)$ were in shock requiring ionotropic support.

\section{Laboratory findings and systemic complications}

K. pneumoniae was isolated from the blood culture in all but one patient (94.74\%). Anterior chamber and vitreous tapping, together with intravitreal antibiotic injection, was arranged for 17 out of 19 patients (89.47\%). Apart from a dry tap, K. pneumoniae was obtained in 6 out of 16 samples. Therefore a positive microbiological yield from a vitreous sample was $37.5 \%$.

Liver abscess was found in 18 patients (94.74\%). For the remaining patient, K. pneumoniae was isolated from the urine sample. The extrahepatic infective foci included empyema (3 patients, $15.79 \%$ ), meningitis and ventriculitis (1 patient, 5.26\%), perianal abscess (1 patient, $5.26 \%$ ), psoas abscess ( 1 patient, $5.26 \%$ ) and skin abscess (1 patient, 5.26\%). 


\section{Management}

Out of the 19 cases, 16 cases received ocular treatment within the day of the consultation. Intravitreal antibiotic injection was arranged for 17 out of 19 patients $(89.47 \%)$ as the primary treatment. The mean number of injection was $1.79 \pm 1.01$ (range $0-6$ ). Pars plana vitrectomy was performed in 3 patients $(15.8 \%)$. Nine patients required evisceration $(47.4 \%)$. The liver abscess was drained under radiological guidance in 14 out of 18 patients $(77.8 \%)$.

\section{Antibiotic sensitivity}

Among the 17 patients who received intravitreal antibiotic injection(s), ceftazidime was used in all 17 patients, vancomycin in 13 patients and amikacin in 9 patients.

All 19 patients received intravenous antibiotics according to the culture sensitivity result and clinical response. The K. pneumoniae strains isolated from the culture were all resistant to ampicillin, while being sensitive to augmentin, cefuroxime, cotrimoxazole, and gentamicin.

\section{Outcome}

The clinical outcome of patients with Klebsiella endophthalmitis is summarized in Figure 1.

Overall, the mortality rate was 4 out of 19 patients (21.1\%). Among those who succumbed from Klebsiella sepsis, the median survival from the first ophthalmic consultation was 29 days (range 8-53 days).

Among the 15 patients who survived the sepsis episode, 9 patients $(60.0 \%)$ required evisceration-one of which required bilateral evisceration. In addition, 3 patients $(20.0 \%)$ had NLP in an involved eye. Only 3 patients $(20.0 \%)$ had a final visual acuity of logMAR 0.30 or above in the better eye.

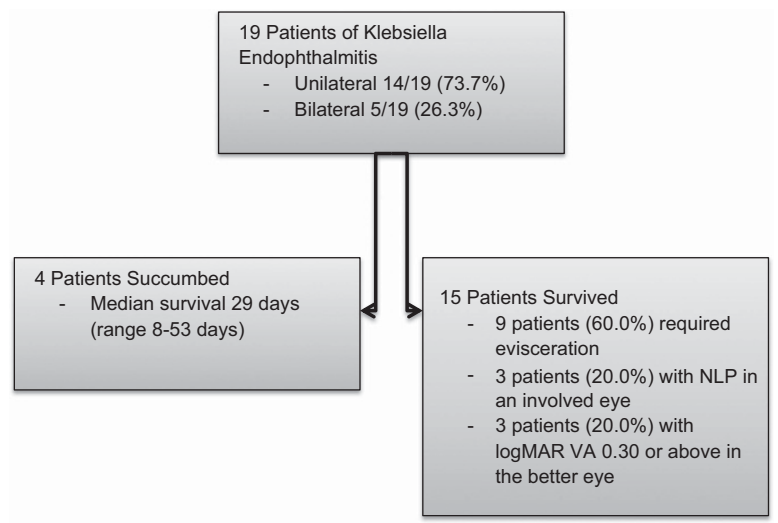

Figure 1 The clinical outcome of patients with Klebsiella endophthalmitis.
Among the patients with bilateral involvement, the respective visual acuity of the two eyes was comparable except in one patient-NLP in the right eye and logMAR 0.10 in the left eye.

\section{Prognostic factors}

The mortality was significantly higher in patients who suffered from septic shock (50.0 vs $0 \%, P=0.018$ ).

Although more patients with disseminated intravascular coagulopathy died in comparison to those without $(40.0$ vs $0 \%$ ), the difference did not reach statistical significance $(P=0.087)$.

A significantly higher proportion of patients presenting with hypopyon required evisceration (85.71 vs $25.00 \%$, $P=0.02)$. Loss of fundal view on presentation, and the presenting visual acuity were not significantly related to evisceration ( $P=0.303, P=0.547$ respectively). However, the patient with the best presenting visual acuity in the series (logMAR 0.40 in both involved eyes) also had the best final visual acuity (logMAR 0.05 both eyes).

Among the 24 eyes involved (14 patients with unilateral involvement and 5 patients with bilateral involvement), 21 eyes had diffuse posterior involvement and 3 eyes had focal posterior involvement. A significantly lower proportion of eyes with diffuse posterior involvement than those with focal posterior involvement had a final visual acuity of logMAR 0.30 or better (4.76 vs $100 \%, P=0.002$ ).

\section{Discussion}

\section{Patient characteristics}

This is the largest series of endogenous endophthalmitis caused by K. pneumoniae in Hong Kong Chinese. Among the 19 patients involved, the mean age was 67.89 years. The infection was community-acquired in all cases. More than half of the patients had underlying diabetes mellitus. This observation was also made in another case series in Singapore, where $46 \%$ of patients had underlying diabetes mellitus. ${ }^{1}$

The mechanism by which diabetes mellitus predisposes to sepsis from K. pneumoniae is still unclear. Although poorly controlled diabetes mellitus itself could result in an immunocompromised state, all the patients in this study were ambulatory before the sepsis episode and clear of an overt immunocompromised state. In fact, current literature also pointed out that Klebsiella endophthalmitis predominantly afflicts otherwise healthy individuals but not those who are overtly immunocompromised, such as those undergoing therapeutic immunosuppression or chemotherapy. ${ }^{11}$

The possible clinical association may be found in our knowledge in the colonization behavior of Klebsiella 
pneumonia. In the study by Chung DR et al, a sampling of 1174 individuals in South Korea showed that

K. pneumoniae was present in the gastrointestinal tract of 248 cases. The gastrointestinal tract, therefore, is the dominant site of colonization. ${ }^{12}$ Infection occurs when the colonizing K. pneumoniae is able to invade through the barriers of the gastrointestinal tract. In poorly controlled diabetes mellitus, autonomic control of the physiology of the gastrointestinal tract may be disturbed. The imbalance between the virulence of the microorganism and host defence mechanisms therefore facilitates the entry of colonizing Klebsiella through the lumen of the gastrointestinal tract into the blood stream. Another possible route of entry is the aspiration of the oropharyngeal colonies of Klebsiella into the respiratory tract. ${ }^{8}$

In this study, liver abscess, as the primary focus of infection, was found in over $90 \%$ of patients with Klebsiella endophthalmitis. However, only $15.8 \%$ of patients had underlying liver diseases such as viral hepatitis or cirrhosis. The close association with liver abscess was also found in the Taiwan case series by Chen et al. ${ }^{3}$ Underlying liver parenchymal and biliary tract pathology, albeit being predisposing factors to liver abscess formation, are uncommon in patients with metastatic Klebsiella infection. ${ }^{13}$ In fact, the incidence of liver abscess in endogenous endophthalmitis caused by K. pneumoniae is much higher than those caused by other micro-organisms. In the case series by Wong et al, ${ }^{1}$ liver abscess was only present in $48 \%$ patients of any cause of endogenous endophthalmitis. The close association between liver abscess and Klebsiella endophthalmitis is compatible with the gastrointestinal colonization of the microorganism, which breaches through host defence mechanisms, enters the portal circulation and multiplies in the liver as the first organ downstream from the bowel.

\section{The hypervirulent klebsiella strain}

The increasing incidence of metastatic infection including endogenous endophthalmis in East Asia has been related to the emergence to a hypervirulent species of K. pneumoniae in this region. ${ }^{7-8}$ Genetic susceptibility among different races has been suggested as a possible reason. ${ }^{14}$

Hypervirulent K. pneumoniae has virulence factors that promote colonization, growth and survival post entry. In a rat subcutaneous abscess model, the growth and survival of hypervirulent $K$. pneumoniae is significantly greater than all four classical K. pneumoniae bacteraemic isolates; resistant to complement-mediated or neutrophilmediated bactericidal activity than $4 / 4$ and $3 / 4$

bacteraemic classical K. pneumoniae isolates,

respectively. ${ }^{11}$ Therefore in non-immune individuals, the primary immune system cannot effectively hold back the infection. Before a specific secondary immune response is mounted a week later, the microorganism has succeeded in causing multiple metastatic infective foci.

\section{Prognosis}

The visual prognosis for Klebsiella endophthalmitis is poor. In this study, nine patients (47.4\%) required evisceration. Among the 15 conscious patients, 13 patients had a final visual acuity of $\mathrm{HM}$ or worse, while 11 patients had NLP in an involved eye. The poor visual outcome is consistent with the findings with the Singaporean case series by Wong JS et al, ${ }_{1}$ in which more than half of the patients had NLP in the involved eye, and few patients regained useful vision.

In this study, some poor visual prognostic indicators were identified. Consistent with previous studies, eyes with diffuse posterior involvement were much less likely than those with focal posterior involvement to attain a final visual acuity of $\log$ MAR 0.30 or better. ${ }^{1,15}$ However, diffuse posterior involvement is a common occurrence in Klebsiella endophthalmitis, accounting for $87.5 \%$ of the involved eyes in this study.

Eyes presenting with hypopyon, as a measure of the severity of intraocular inflammation, were also more likely to require evisceration. During bacteraemia, the microorganism metastasizes to the vascular choroid. If the host defence is breached, the microorganism is spilled over through the retina to the vitreous gel. The amount of inflammatory cells and debris has to be substantial in order to be able to get through the zonules and collect in the anterior chamber, leading to hypopyon. ${ }^{16}$

Systemically, Klebsiella endophthalmitis is associated with a high mortality. The overall mortality rate in this study was $21.1 \%$-higher than the quoted mortality rate of $6-14 \%$ of Klebsiella liver abscesses in the literature. ${ }^{17}$ In this study, patients with Klebsiella endophthalmitis characteristically had a high incidence of disseminated intravascular coagulopathy (52.6\%) and septic shock $(42.1 \%)$. Although both of these factors are indicators of systemic inflammatory response, only septic shock was significantly associated with a higher mortality. After all, spontaneous hemorrhage due to disseminated intravascular coagulopathy is uncommon as the bleeding tendency can be readily corrected with transfusion.

On the other hand, haemodynamic instability affects the perfusion of all the essential organs in the body and is more difficult to control even with inotropes.

\section{Management recommendations}

Universal screening. In contrast to the classical symptomatology of endophthalmitis, exemplified by blurring of vision, eye pain, redness and floaters, Klebsiella 
endophthalmitis may present with only subtle symptoms. In this study, only less than half of the patients presented with blurring, and conjunctival redness was not observed in more than $20 \%$ of patients. The visual symptoms were also under-reported in patients had impaired consciousness due to septic shock. Given the high tendency of metastatic ocular infection, universal screening by the ophthalmologist is recommended for all patients with Klebsiella sepsis.

The use of antibiotics. Liver abscesses caused by extended spectrum $\beta$-lactamase-producing K. pneumoniae are uncommon. ${ }^{18}$ In this study, all the Klebsiella isolates were only resistant to ampicillin. Antibiotics such as ampicillinsulbactam, a third-generation cephalosporin, aztreonam or quinolone can be used. In particular, third-generation cephalosporin is recommended due to its good penetration in the vitreous cavity and cerebral spinal fluid. Peak vitreous concentrations of at least $2 \mathrm{mg} / 1 \mathrm{can}$ be achieved. ${ }^{19}$ In case of extended spectrum $\beta$-lactamaseproducing strains, imipenem and meropenem can be used. ${ }^{20}$

The role of intravitreal antibiotic injection is supported by the high yield of K. pneumonie from vitreous tapping samples in this study (37.5\%). Many of the involved eyes of Klebsiella endophthalmitis were still not rendered sterile despite the administration of systemic antibiotics.

Drainage of the primary focus of infection. Adherent to the basic tenets of the treatment of infection, all abscesses or closed compartment infection should be drained. In patients with Klebsiella endophthalmitis, drainage of the liver abscess should be arranged early where appropriate. In this study, the value of liver abscess drainage is specifically illustrated by patient WH (Table 1) who had an inadequately managed liver abscess and presented with endogenous endophthalmitis only during the second episode of bacteraemia.

In this study, radiologically guided percutaneous drainage was performed for all patients. However, the hyperviscomuous property of hypervirulent K. pneuomoniae may potentially impede continuous drainage through the catheter. Open surgical drainage may have a role in these cases. However, the unstable haemodynamic state and bleeding tendency of these patients may limit the choice of intervention. ${ }^{8}$

The value of early vitrectomy. The benefit of early vitrectomy is still controversial for endogenous endophthalmitis. There are studies in favor of early vitrectomy. ${ }^{21-24}$ However, the benefit could not be replicated in other studies. ${ }^{1,25}$

In this regard, we advocate early vitrectomy for patients with poor visual prognostic indicators especially the presence of hypopyon, which is independently associated with a higher rate of evisceration in this study. The biological rationale for early vitrectomy is two-fold. First of all, vitrectomy can rapidly reduce the bacterial load and inflammatory mediators, which can lead to devastating tissue damage, in the vitreous cavity. ${ }^{14}$ Secondly, when the dense vitreous abscess is removed, diffusion of antibiotics from the blood stream to the vitreous cavity is facilitated. ${ }^{26}$ Evisceration should be reserved for patients with painful blind eyes. However, aggressive surgical intervention may be limited by the haemodynamic instability and bleeding tendency of patients with Klebsiella sepsis.

\section{Conclusion}

The ophthalmologist should view endogenous endophthalmitis due to K. pneumoniae as being part of an invasive syndrome caused by the microorganism. A multi-disciplinary approach involving close liaison with physicians and microbiologists is essential. Apart from ophthalmic management, systemic control of sepsis with the right antibiotics and drainage of the primary abscess are equally important. Nevertheless, the prognosis of this disease remains poor both visually and systemically due to the virulence of K. pneumoniae.

\section{Summary}

What was known before

- Klebsiella endophthalmitis is associated with poor prognosis.

What this study adds

- The prognostic factors of Klebsiella endophthalmitis: hypopyon and diffuse posterior involvement (poor visual outcome) and septic shock (mortality).

\section{Conflict of interest}

The authors declare no conflict of interest.

\section{Acknowledgements}

We did not receive any sponsorship or financial support from any academic or commercial organization.

\section{References}

1 Wong JS, Chan TK, Lee HM, Chee SP. Endogenous bacterial endophthalmitis: an East Asian experience and a reappraisal of a severe ocular affliction. Ophthalmology 2000; 107: 1483-1491. 
2 Schiedler V, Scott IU, Flynn HW, Davis JL, Benz MS, Miller D. Culture-proven endogenous endophthalmitis: clinical features and visual outcomes. Am J Ophthalmol 2004; 137: 725-731.

3 Chen YJ, Kuo HK, Wu PC, Kuo ML, Tsai HH, Liu CC et al. A 10-year comparison of endogenous endophthalmitis outcomes: an east Asian experience with Klebsiella infection. Retina 2004; 24: 383-390.

4 Ness T, Pelz K, Hansen LL. Endogenous endophthalmitis: microorganisms, disposition and prognosis. Acta Ophthalmol Scand 2007; 85: 852-856.

5 Chang FY, Chou MY, Fan RL, Shaio MF. A clinical study of Klebsiella liver abscess. Taiwan Yi Xue Hui Za Zhi 1988; 87: 282-287.

6 Chang FY, Chou MY. Comparison of pyogenic liver abscesses caused by Klebsiella pneumoniae and non- $K$ pneumoniae pathogens. J Formos Med Assoc 1995; 94: 232-237.

7 Siu LK, Yeh KM, Lin JC, Fung CP, Chang FY. Klebsiella pneumoniae liver abscess: a new invasive syndrome. Lancet Infect Dis 2012; 12: 881-887.

8 Shon AS, Bajwa RPS, Russo TA. Hypervirulent (hypermucoviscous) Klebsiella pneumonia: a new and dangerous breed. Virulence 2013; 4(2): 107-118.

9 Endophthalmitis Vitrectomy Study Group. Results of the Endophthalmitis Vitrectomy Study. A randomized trial of immediate vitrectomy and of intravenous antibiotics for the treatment of postoperative bacterial endophthalmitis. Arch Ophthalmol 1995; 113: 1479-1496.

10 Lange C, Feltgen N, Junker B, Shulze-Bonsel K, Bach M. Resolving the clinical acuity categories 'hand motion' and 'counting fingers' using the Freiburg Visaul Acuity Test (FrACT). Graefes Arch Clin Exp Ophthalmol 2009; 247(1): 137-142.

11 Pomakova DK, Hsiao CB, Beanan JM, Olson R, MacDonald U, Keynan $\mathrm{Y}$ et al. Clinical and phenotypic differences between classic and hypervirulent Klebsiella pneumoniae: an emerging and under-recognized pathogenic variant. Eur J Clin Microbiol Infect Dis 2012; 31 (6):981-989.

12 Chung DR, Lee H, Park MH, Jung SI, Chang HH, Kim YS et al. Fecal carriage of serotype K1 Klebsiella pneumoniae ST23 strains closedly related to liver abscesses isolates in Koreans living in Korea. Eur J Clin Microbiol Infect Dis 2012; 31: 481-486.

13 Wang JH, Liu YC, Lee SS, Yen MY, Chen YS, Wang JH et al. Primary liver abscess due to Klebsiella pneumoniae in Taiwan. Clin Infect Dis 1998; 26: 1434-1438.
14 Lee S, Um T, Joe SG, Hwang JU, Kim JG, Yoon YH et al. Changes in the clinical features and prognostic factors of endogenous endophthalmitis: fifteen years of clinical experience in Korea. Retina 2012; 32: 977-984.

15 Lee SY, Chee SP. Group B Streptococcus endogenous endophthalmitis. Case reports and review of the literature. Ophthalmology 2002; 109(10): 1879-1886.

16 Ang M, Jap A, Chee SP. Prognostic factors and outcomes in endogenous Klebsiella pneumoniae endophthalmitis. Am J Ophthalmol 2011; 151: 338-344.

17 Cerwenka H. Pyogenic liver abscess: differences in etiology and treatment in Southeast Asia and Central Europe. World J Gastroenterol 2010; 16(20): 2458-2462.

18 Lin YT, Jeng YY, Chen TL, Fung CP. Bacteremic communityacquired pneumonia due to Klebsiella pneumoniae: clinical and microbiological characteristics in Taiwan, 2001-2008. BMC Infect Dis 2010; 10: 307.

19 Sharir M, Triester G, Kneer J, Rubinstein E. The intravireal penetration of ceftriaxone in man following systemic administration. Invest Ophthalmol Vis Sci 1090; 30: 2179-2183.

20 Cheng HP, Siu LK, Chang FY. Extended-spectrum cephalosporin compared to cefazolin for treatment of Klebsiella pneumoniae-caused liver abscess. Antimicrob Agents Chemother 2003; 47: 2088-2092.

21 Connell PP, O'Neill EC, Fabinyi D, Islam FM, Buttery R, McCombe $\mathrm{M}$ et al. Endogenous endophthalmitis: 10-year experience at a tertiary referral centre. Eye (Lond) 2011; 25: 66-72.

22 Yoon YH, Lee SU, Sohn JH, Lee SE. Result of early vitrectomy for endogenous Klebsiella pneumoniae endophthalmitis. Retina 2003; 23: 366-370.

23 Ishii K, Hiraoka T, Kaji Y, Sakata N, Motoyama Y, Oshika T. Succesful treatment of endogenous Klebsiella pneumoniae endophthalmitis: a case report. Int Ophthalmol 2011; 31: 29-31.

24 Itoh M, Ikewaki J, Kimoto K, Itoh Y, Shinoda K, Nakatsuka K. Two cases of endogenous endophthalmitis caused by grampositive bacteria with good visual outcome. Case Rep Ophthalmol 2010; 1: 56-62.

25 Hwang JH, Cho NC. Prognostic factors in patients with endogenous endophthalmitis. J Korean Ophthalmol Soc 2009; 50: 858-863.

26 Lim HW, Shin JW, Cho HY, Kim HK, Kang SW et al. Endogenous endophthalmitis in the Korean population: a six-year retrospective study. Retina 2014; 34: 592-602. 